\title{
Adiposidad corporal y niveles de actividad física en adolescentes Body adiposity and levels of physical activity in adolescents
}

*Marco Antonio Cossio-Bolaños, **Ruben Vidal-Espinoza, ***Marcelo Pino-Valenzuela, ****Cristian Luarte-Rocha, *****Margot Rivera-Portugal, ******Jose Sulla-Torres, *Rossana Gomez-Campos

*Universidad Católica del Maule Chile), **Universidad Catolica Silva Henriquez (Chile), ***Universidad Santo Tomás (Chile), ****Universidad San Sebastian (Chile), *****Universidad Nacional de San Agustín de Arequipa (Perú), ******Universidad Católica de Santa Maria (Perú)

Resumen. Los objetivos del estudio son comparar los niveles de adiposidad corporal expresado por medio de Índice de Masa Corporal IMC y circunferencia de la cintura CC con la referencia americana del Centro para el Control y la Prevención de Enfermedades CDC-2012 y analizar si estos niveles de adiposidad corporal difieren entre los adolescentes, según categorías de actividad física AF. Se efectuó un estudio descriptivo transversal en 765 adolescentes (432 hombres y 333 mujeres). La selección de la muestra fue no-probabilística (cuotas). El rango de edad oscila de 10.0 a 18,9 años. Se evaluó el peso, la estatura, CC y se calculó el IMC. Se aplicó un cuestionario de AF. No hubo diferencias significativas de IMC y CC con la referencia del CDC-2012 en adolescentes de ambos sexos (p<0.05). Se observó prevalencia de bajo nivel AF (5,3\% para hombres y 14,7\% para mujeres), en moderada $\mathrm{AF}$ ( $47.7 \%$ en hombres y $63,4 \%$ mujeres) y con un elevada $\mathrm{AF}$ (47,0\% en hombres y 21,9\% en mujeres). Se observó relación positiva entre los IMC y CC en las tres categorías (bajo nivel: $r=0,94$ hombres y $r=0,87$ mujeres, moderado nivel: $r=0,87$ hombres y $r=0,86$ mujeres y elevado nivel de AF: $r=0,85$ hombres y r= 0,63 mujeres). Los adolescentes estudiados reflejan similares patrones de adiposidad corporal que la referencia del CDC-2012. Además, los clasificados con elevada AF, reflejaron menor adiposidad abdominal que las demás categorías de AF.

Palabras clave: Actividad física, adolescentes, Adiposidad corporal.

Abstract. The objectives of the study were to compare body adiposity levels expressed by means of Body Mass Index (BMI) and waist circumference (WC) with the American reference of the Center for Disease Control and Prevention (CDC-2012); and to analyze whether these body adiposity levels differ among adolescents by physical activity categories. A cross-sectional descriptive study was carried out with 765 adolescents ( 432 boys and 333 girls). The selection of the sample was non-probabilistic (quotas). We included 4 schools from the city of Talca, Chile. The age interval ranged from 10.0 to 18.9 years old. Weight, height, and WC were evaluated, and BMI was calculated. A PA questionnaire was applied. There were no significant differences in BMI and WC with the reference of CDC-2012 in adolescents of both genders ( $\mathrm{p}<0.05$ ). Prevalence of low PA level was observed ( $5.3 \%$ for boys, and $14.7 \%$ for girls), as well as moderate PA level (47.7\% in boys, and 63.4\% girls) and high PA level (47,0\% in boys, and $21.9 \%$ in girls). Positive relationship was found between the indicators of body adiposity (BMI and WC) in the three categories (low level: $r=0.94$ boy, and $r=0.87$ girls; moderate level: $r=0.87$ boys, and $r=0.86$ girls; and high level of PA: $r=0.85$ boys, and $r=0.63$ girls). The adolescents in this study reflect similar patterns of corporal adiposity as in the CDC-2012 for both genders. In addition, schoolchildren classified with high level of PA reflected lower abdominal adiposity than those in the other PA categories.

Keywords: Physical activity, adolescents, body adiposity.

\section{Introducción}

La prevalencia del sobrepeso y la obesidad es cada vez de mayor preocupación en la salud publica en diversos lugares del mundo (Afshin et al., 2017; Ogden et al., 2006) y específicamente en chile, donde en la última encuesta nacional de salud (Ministerio de Salud, 2018), utilizando como indicador el Índice de Masa Corporal IMC detectó 39,8\% de sobrepeso y 31,2 de obesidad.

Actualmente existe evidencias científicas que han demostrado que los estilos de vida perjudican los niveles de adiposidad corporal por medio de diversos factores de comportamiento sedentarios (Carson, Hunter, et al., 2016). Estos patrones, independientemente de la región geográfica y cultural, los niños y adolescentes a lo largo de todas las etapas de la vida van adquiriendo diversos niveles de actividad física (Poitras et al., 2016), deficiencias en la alimentación (Sahoo et al., 2015), insuficiente sueño (Carson, Tremblay, Chaput, \& Chastin, 2016), entre otros factores claves, los que pueden contribuir a la presencia de la epidemia de obesidad a edades cada vez más tempranas.

Fecha recepción: 13-05-19. Fecha de aceptación: 08-08-19 Marco Antonio Cossio-Bolaños mcossio1972@hotmail.com
En general, una de las técnicas que se suele utilizar para evaluar la adiposidad corporal son las variables antropométricas, cuyos índices reflejan la relación entre variables que dan como resultados el IMC, la circunferencia de la cintura CC, índice cintura/estatura, cintura/cadera (Vargas et al., 2014), entre otros indicadores.

Por el contrario, en relación a los patrones de Actividad Física AF, hasta donde se sabe, no existe consenso definido sobre un método especifico que mida los niveles de AF en niños y adolescentes chilenos, a pesar de que algunos estudios en los últimos años han mostrado interés por el uso de técnicas objetivas (Godard et al., 2016; Godard, Román, Rodríguez, Leyton, \& Salazar, 2012) y subjetivas (Yáñez-Silva, Hespanhol, Gómez-Campos, \& Cossio-Bolaños, 2014).

En general, independientemente del tipo de método a utilizar, la AF regular de moderada a eleva intensidad se considera como medida preventiva para la eficiencia de la aptitud cardiorrespiratoria (Poitras et al., 2016) y para mantener un adecuado estado de salud. Además, comportamientos sedentarios y la presencia de exceso de grasa corporal aumentan el riesgo de padecer enfermedades cardiovasculares y metabólicas (World Health Organization, 2018), por lo que elevados niveles de AF confieren protección contra las enfermedades crónicas en individuos que presentan factores de riesgo metabólicos (Brunet, Chaput, \& Tremblay, 2007). 
En esencia, monitorear continuamente las tendencias observables de los patrones de AF y la adiposidad corporal, pueden permitir el desarrollo y la implementación de Políticas de salud a nivel escolar. Esta información, es relevante, puesto que puede contribuir a sensibilizar sobre los hábitos saludables en edades escolares, a la manutención del estado de peso dentro de la categoría de normo-peso según edad y sexo y para promocionar la práctica de la AF en grupos con exceso de adiposidad corporal.

Por lo tanto, este estudio inicialmente tiene como objetivo comparar los niveles de adiposidad corporal expresado por medio de IMC y CC con la referencia americana del Centro para el Control y la Prevención de Enfermedades CDC2012 (Fryar, Gu, \& Ogden, 2012), y, en segundo lugar, analizar si estos niveles de adiposidad corporal difieren entre los adolescentes, cuando son clasificados por niveles AF. Para ello, esta investigación utilizó una técnica subjetiva para clasificar los niveles de AF entre los adolescentes investigados.

\section{Metodología}

\section{Tipo y de estudio y muestra}

Se efectuó un estudio descriptivo transversal en 765 adolescentes (432 hombres y 333 mujeres). El cálculo de la muestra fue no-probabilística (cuotas). Se estudiaron 4 Colegios de la Ciudad de Talca, Chile. Estos Colegios son Municipales. El rango de edad oscila de 10.0 a 18,9 años.

Se incluyeron en el estudio a los adolescentes que presentaban 10 a 18,9 años y que podían leer y comprender el cuestionario aplicado. Se retiró a los que no completaron las mediciones antropométricas y el cuestionario aplicado. Además, se retiró a los alumnos cuyos padres no autorizaron la evaluación a sus hijos. Todo el desarrollo del estudio estuvo resguardado por el comité de ética de la Universidad Católica del Maule.

\section{Procedimientos}

Se solicitó permiso a la Dirección de cada Colegio para evaluar la antropometría y la aplicación del cuestionario de AF. La recolección de datos se desarrolló en horario escolar (8:30 a 13:00 horas) y de lunes a viernes durante los meses de agosto a noviembre del 2018. Todo el protocolo estuvo a cargo de 4 evaluadores altamente entrenados y con amplia experiencia en técnicas antropométricas y de encuestas.

La antropometría se evaluó en el departamento de educación física de cada colegio. Se utilizó el protocolo estandarizado de (Ross, Marfell-Jones, MacDougall, Wenger, \& Green, 1991). Este procedimiento estuvo a cargo de dos evaluadores. Se consideró tener en cuenta durante la evaluación que los participantes sean medidos sin zapatos, con short y polera El peso corporal (kg) fue evaluado utilizando una balanza electrónica (Tanita, Reino Unido), con una escala 0 a $150 \mathrm{~kg}$, y con precisión de 100 g. La estatura de pie se evaluó mediante un estadiómetro portátil (Seca Gmbh \& Co. KG, Hamburgo, Alemania) con una precisión de 0,1 mm. La CC se evaluó con una cinta métrica Seca de metal y graduada en milímetros con una precisión de $0,1 \mathrm{~cm}$. Se calculó el índice de Masa Corporal IMC [IMC $(\mathrm{kg} / \mathrm{m} 2)=$ peso/estatura $\left.{ }^{2}\right]$.

La actividad física AF, se evaluó utilizando la técnica de la encuesta. Se aplicó el instrumento propuesto por (GómezCampos, Vilcazán, Arruda, Hespañol, \& Cossio-Bolaños, 2012). El cuestionario fue aplicado en las salas según curso y horarios de las clases de educación física. Tuvo una duración de 10 minutos. Se aplico de forma tradicional a lápiz y papel. Un evaluador explicó el procedimiento, mientras que un segundo resolvía algunas dudas de los alumnos.

La valoración de los niveles de AF se basaron según puntos de corte adoptado por (Cossío-Bolaños et al., 2017). $<$ P15 bajo nivel de AF, P15-P85 moderado nivel de AF y >P85 elevado nivel de AF.

\section{Estadística}

La normalidad de datos se verificó por medio de Kolmogórov-Smirnov (K-S). Se calculó la estadística descriptiva de media, desviación estándar, frecuencias y porcentajes. Las comparaciones con la referencia y entre géneros se verifico por medio de test t para muestras independientes. Las diferencias de prevalencia fueron analizadas por medio chi cuadrado $\left(\mathrm{X}^{2}\right)$. Las comparaciones entre los niveles de AF se verificaron por Anova de una vía y pos Hoc Tukey. Las relaciones bi-variadas entre variables se verificaron por medio del coeficiente de Spearman. En todos los casos se adoptó una probabilidad $\mathrm{p}<0.05$. Los cálculos se efectuaron en Sigma Estat 8.0.

\section{Resultados}

Las variables antropométricas, adiposidad corporal y patrones de actividad física se observan en la tabla 1. Los hombres presentaron mayor peso corporal, estatura, niveles de actividad física (tipo y duración) en relación a las mujeres $(\mathrm{p}<0.05)$. En cuanto a la prevalencia de los niveles de AF se observó diferencias significativas entre las categorías en ambos sexos. En Hombres el 23\% y en mujeres el 9,4\% reflejaron bajos niveles de AF.

\begin{tabular}{|c|c|c|c|c|c|c|}
\hline \multirow[b]{2}{*}{ Variables } & \multicolumn{2}{|c|}{ Hombres $(\mathrm{n}=432)$} & \multicolumn{2}{|c|}{ Mujeres $(n=333)$} & \multicolumn{2}{|c|}{ Ambos $(n=765)$} \\
\hline & $\mathrm{x}$ & $\mathrm{DE}$ & $\mathrm{X}$ & $\mathrm{DE}$ & $\mathrm{X}$ & $\mathrm{DE}$ \\
\hline Edad (años) & 15 & 2,7 & 14,7 & 2,9 & 14,9 & 2,8 \\
\hline \multicolumn{7}{|l|}{ Antropometría } \\
\hline Peso (kg) & 61,7 & 15,6 & $56,6^{*}$ & 14,5 & 59,5 & 15,3 \\
\hline Estatura $(\mathrm{cm})$ & 163,9 & 13,1 & $155,4 *$ & 9,6 & 160,3 & 12,4 \\
\hline IMC $\left(\mathrm{kg} / \mathrm{m}^{2}\right)$ & 22,6 & 4,0 & 23,2 & 4,6 & 22,9 & 4,3 \\
\hline C. Cintura (cm) & 54 & 25,7 & 53,9 & 24,6 & 53,9 & 25,2 \\
\hline \multicolumn{7}{|c|}{ Actividad Física (Puntos) } \\
\hline Tipo & 10,2 & 3,2 & $8,4^{*}$ & 2,8 & 9,4 & 3,1 \\
\hline Frecuencia & 5,9 & 1,7 & 5,2 & 1,6 & 5,6 & 1,7 \\
\hline Duración & 9,1 & 2,6 & $7,7^{*}$ & 2,8 & 8,5 & 2,8 \\
\hline Intensidad & 7,4 & 2,0 & 6,6 & 1,8 & 7,1 & 2,0 \\
\hline Total de AF & 32,6 & 7,8 & $27,9 *$ & 7,3 & 30,6 & 7,9 \\
\hline Prevalencia nivel AF & $f$ & $\%$ & $F$ & $\%$ & $f$ & $\%$ \\
\hline Bajo & 23 & 5,3 & 49 & 14,7 & 72 & 9,4 \\
\hline Medio & 206 & 47,7 & 211 & 63,4 & 417 & 54,5 \\
\hline Elevado & 203 & 47 & 73 & 21,9 & 276 & 36,1 \\
\hline
\end{tabular}

Leyenda: AF: Actividad Física, IMC: Índice de Masa Corporal, DE: Desviación Estándar, C: Circunferencia, *: Diferencia significativa ( $\mathrm{p}<0.05), \mathrm{X}^{2}=16.24$ (gl:4, $\left.\mathrm{p}=0.0027\right)$, f: Frecuencia.

En las comparaciones de adiposidad corporal (IMC y CC), los adolescentes estudiados reflejaron similares patrones de IMC y CC en todos los rangos de edad y en ambos sexos. Los valores medios se incrementan conforme la edad aumenta.

En la figura 2 se muestra los valores de adiposidad corporal según los niveles de AF. Los hombres clasificados con alto nivel de AF, mostraron valores inferiores de IMC respecto a los de moderada y bajo nivel de AF ( $\mathrm{p}<0.05)$, mientras que en las mujeres no hubo diferencias de IMC en las tres 
Figura 1. Comparación de la adiposidad corporal (IMC y CC) de los adolescentes estudiados con la referencia americana del CDC-2012.

categorías. En cuanto a la CC, tanto en hombres, como en mujeres los valores de CC son significativamente inferiores frente a los clasificados como moderado y bajo nivel de AF $(p<0.05)$.

Figura 2. Comparación de la adiposidad corporal (IMC y CC) en ambos sexos, según niveles de actividad física.

Las relaciones entre indicadores de adiposidad corporal (IMC-CC) en cada categoría de AF se observan en la figura 3. Se observa que, en ambos sexos, las correlaciones de IMC con CC van disminuyendo a medida que se realza mayor AF. Esto indica que la relación de adiposidad corporal en los sujetos que realizan elevado nivel de AF es más débil que los clasificados en las otras categorías (moderado y bajo niel de $\mathrm{AF})$.

Figura 3. Relación bi-variada entre IMC y CC en escolares de ambos sexos clasificados con bajo, moderado y elevado nivel de AF.

\section{Discusión}

Los resultados del estudio han mostrado que los patrones de adiposidad corporal, tanto, por IMC y CC son similares con la referencia de CDC-2012 (Fryar et al., 2012). Estos valores son relativamente similares a otros estudios efectuados en Chile (Gómez-Campos et al., 2015; Vargas et al., 2014). De hecho, estos hallazgos expresados en términos de IMC y CC, por lo general señalan que los parámetros de adiposidad van aumentando conforme la edad avanza por efectos del crecimiento físico (Gomez-Campos et al., 2016) y en mayor proporción debido a los bajos niveles de AF que los jóvenes vienen atravesando, en especial en estos tiempos, en el que el sobrepeso y la obesidad va en aumento en chile, no solo, en adultos, sino también en niños y adolescentes (Gatica Mandiola et al., 2013).

En ese contexto, es necesario monitorear permanentemente los parámetros corporales en niños y adolescentes para evitar futuramente la acumulación de exceso de grasa corporal (Myrtaj et al., 2018) y en especial para la prevención de enfermedades y la promoción de la salud (Ahn, Choi, \& Sohn, 2013).

Pues es ampliamente conocido que el aumento de nivel de la adiposidad se debe sin duda, a la adopción temprana de los modernos estilos de vida, a través del cual, alcanzan mayor IMC y perímetro de la cintura (Wardle \& Boniface, 2008), además presentan elevado riesgo de desarrollar diabetes mellitus, hipertensión arterial, enfermedad arterial coronaria, síndrome metabólico (Lee, Chung, Kang, \& Yu, 2012) e inclusive se asocia con variables psicológicas como la depresión, entre otras (Murabito, Massaro, Clifford, Hoffmann, \& Fox, 2013). Por el contrario, adolescentes clasificados como normales o bajo peso a menudo presentan mayor fuerza muscular e inclusive son grupos físicamente más activos (Sepúlveda Cáceres et al., 2018; Gomez-Campos et al., 2019).

En ese sentido, como segundo objetivo, este estudio buscó determinar las diferencias de adiposidad corporal según categorías de AF. Los resultados han demostrado que un 9,4\% se encuentra clasificado con bajo nivel de AF, 54.5\% con moderado nivel de AF, y 36.1\% con elevado nivel de AF.

En general, los adolescentes de ambos sexos clasificados con elevados niveles de AF evidenciaron bajos niveles de adiposidad (IMC y CC) en relación a sus contrapartes que realizaban bajos y moderados niveles de AF. Este patrón demuestra que los adolescentes que realizan elevados niveles de AF para su edad y sexo reflejaron valores inferiores de adiposidad corporal. Estos hallazgos confirman que la práctica de la AF contribuye a un correcto desarrollo de los huesos, músculos y articulaciones, así como la tenencia de un sistema cardiovascular saludable y ayudar a tener un peso óptimo (Zurita-Ortega et al., 2018).

Esto significa que la continua práctica de actividad física es un aliciente constante y cotidiano en nuestra vida diaria, debido a los múltiples beneficios en la salud física y mental (Aznar Lain \& Webster, 2006). Pues en los últimos años, las comunidades urbanas, han sufrido una rápida evolución del estilo de vida, los que han ido produciendo algunos cambios socioculturales que pueden estar afectando los niveles de AF de niños y adolescentes (Gomes-Mascarenhas et al., 2005). Este patrón incrementa los niveles de adiposidad a edades cada vez más tempranas.

De hecho, promover la actividad física es una estrategia esencial en materia de salud pública, que favorece el mantenimiento y mejora de la salud en la población general (Cervantes, Amador-Rodero, \& Arrázola-David, 2017) y específicamente en los centros escolares de chile. Por lo que, de acuerdo a los resultados obtenidos en este estudio, no es suficiente AF moderada, siendo necesario desarrolla de al 
parecer de elevada intensidad.

En consecuencia, la cantidad óptima de AF en la adolescencia requiere abordar un sinnúmero de retos científicos, ya que los resultados pueden variar entre los métodos utilizados (objetivos y/o subjetivos) y los tipos de población investigadas (urbanos y/o rurales), aunque la promoción de la AF debe comenzar a edades tempranas, especialmente en los colegios, donde las clases de educación física y el tiempo de recreo son clave para la promoción de AF en el ámbito escolar, ya que niños y adolescentes permanecen entre seis y ocho horas diarias en el colegio (Wechsler, Devereaux, Davis, \& Collins, 2000).

En consecuencia, la realización de AF supone hoy en día una forma de desarrollo del potencial personal, que favorece la ampliación de todas las facetas de la personalidad humana (Gentil Adarve et al, 2019), como, por ejemplo, mejorarlos niveles de aptitud física, la composición corporal, la salud ósea, cardiovascular, salud mental, entre otros aspectos. Aunque también al parecer es necesario llevar a cabo una serie de intervenciones con la finalidad de concientizar a los niños sobre la vida sedentaria y poco saludable que vienen llevando acabo (Núñez-Quiroga et al, 2019).

Esta investigación presenta algunas limitaciones que deben ser señaladas y deben ser consideradas para futuros estudios. No fue posible recabar información de las horas y la cantidad de días que realizaban educación física e inclusive de la modalidad de la práctica deportiva. Estas variables podrían sesgar los resultados obtenidos y originar discrepancias al contrastar con otros estudios nacionales e internacionales. Aunque, por el contrario, por la cantidad de sujetos, y el tipo de instrumento utilizado para valorar la AF podría ser una ventaja categorizar a los adolescentes de forma rápida en niveles. Esto es una ventaja para ser aplicado en el ámbito escolar, dado que se necesita monitorear a grandes poblaciones en periodos cortos de tiempo.

\section{Conclusión}

Este estudio, concluye que los adolescentes estudiados reflejan similares patrones de adiposidad corporal que la referencia del CDC-2012 en ambos sexos. Además, los adolescentes clasificados con elevado nivel de AF, reflejaron menor adiposidad abdominal que los categorizados con moderado y bajo nivel de adiposidad. Estos resultados sugieren que los jóvenes clasificados con moderado y bajo nivel de AF deben de realizar programas de AF con suma urgencia.

\section{Agradecimientos}

Al proyecto interno UCM 434215.

\section{Referencias}

Afshin, A., Forouzanfar, M. H., Reitsma, M. B., Sur, P., Estep, K., Lee, A., ... Murray, C. J. L. (2017). Health Effects of Overweight and Obesity in 195 Countries over 25 Years. The New England Journal of Medicine, 377(1), 13-27. https://doi.org/10.1056/NEJMOA1614362

Ahn, Y., Choi, S., \& Sohn, M. (2013). Adiposity of Korean school-age children measured by national and international growth charts. Research in Nursing \& Health, 36(1), 16-25. https://doi.org/10.1002/nur.21510

Aznar Lain, S., \& Webster, T. (2006). Actividad física y salud en la infancia y la adolescencia. Guía para todas las personas que participan en su educación - Publicaciones - Ministerio de Educación, Cultura y Deporte. (C. de I. y D. educativa Ministerio de Educacion y Cultura, Ed.) (1ra ed.). Madrid, España. https://doi.org/10.4438/ 351-06-042-1

Brunet, M., Chaput, J.-P., \& Tremblay, A. (2007). The association between low physical fitness and high body mass index or waist circumference is increasing with age in children: the 'Québec en Forme' Project. International Journal of Obesity, 31(4), 637-643. https://doi.org/ 10.1038/sj.ijo.0803448

Carson, V., Hunter, S., Kuzik, N., Gray, C. E., Poitras, V. J., Chaput, J.-P., ... Tremblay, M. S. (2016). Systematic review of sedentary behaviour and health indicators in schoolaged children and youth: an update. Applied Physiology, Nutrition, and Metabolism, 41(6 (Suppl. 3)), S240-S265. https://doi.org/10.1139/apnm-2015-0630

Carson, V., Tremblay, M. S., Chaput, J.-P., \& Chastin, S. F. M. (2016). Associations between sleep duration, sedentary time, physical activity, and health indicators among Canadian children and youth using compositional analyses. Applied Physiology, Nutrition, and Metabolism, 41(6 (Suppl. 3)), S294-S302. https://doi.org/ 10.1139/apnm-2016-0026

Cervantes, K., Amador-Rodero, E., \& Arrázola-David, M. (2017). Nivel de actividad física en niños de edades de 6 a 12 años en algunos colegios de Barranquilla-Colombia, en el año 2014-2015. Biociencias, 12(1), 17-23. Retrieved from https://dialnet.unirioja.es/servlet/ articulo?codigo $=6228794$

Cossío-Bolaños, M., Méndez-Cornejo, J., Luarte Rocha, C., Vargas-Vitoria, R., Canqui-Flores, B., \& Gomez-Campos, R. (2017). Patrones de actividad física de adolescentes escolares: validez, confiabilidad y propuesta de percentiles para su evaluación. Revista Chilena de Pediatría, 88(1), 73-82. https://doi.org/10.1016/ j.rchipe.2016.07.010

Fryar, C. D., Gu, Q., \& Ogden, C. L. (2012). Anthropometric reference data for children and adults: United States, 20072010. Vital and Health Statistics. Series 11, Data from the National Health Survey, (252), 1-48. Retrieved from http://www.ncbi.nlm.nih.gov/pubmed/25204692

Gatica Mandiola, P., Vargas Vitoria, C. R., Jirón Amaro, O., Herrera Blanco, M., Duarte Farfán, C., Gómez Campos, R., ... Cossio-Bolaños, M. (2013). Cambios en la adiposidad corporal de adolescentes escolares (1997-2007). Nutrición Clínica y Dietética Hospitalaria, 33(3), 2329. https://doi.org/10.12873/333bodyfat

GentilAdarve, M., Zurita Ortega, F., Gómez Sánchez, V., PadialRuz, R., \& Lara-Sánchez, A. (2019). Influencia de la práctica de actividad física en el auto-concepto de adolescentes. Retos: Nuevas Perspectivas de Educacioìn Fiìsica, Deporte y Recreacioìn, 36, 342-347.

Godard, C., Carlin, L., Torres, J., Rodríguez, M. del P., Leyton, B., \& Salazar, G. (2016). Nivel de intensidad de las principales actividades físicas de escolares chilenos: determi- 
nación por acelerometría. Revista Chilena de Nutrición, 43(3), 3-3. https://doi.org/10.4067/S071775182016000300003

Godard, C., Román, M., Rodríguez, M. D. P., Leyton, B., \& Salazar, G. (2012). Variabilidad de la actividad física en niños chilenos de 4 a 10 años: estudio por acelerometría. Archivos Argentinos de Pediatria, 110(5), 388-393. https://doi.org/10.5546/aap.2012.388

Gómez-Campos, R., Andruske, C. L., Hespanhol, J., Torres, J. S., Arruda, M., Luarte-Rocha, C., \& Cossio-Bolaños, M. (2015). Waist Circumferences of Chilean Students: Comparison of the CDC-2012 Standard and Proposed Percentile Curves. International Journal of Environmental Research and Public Health, 12(7), 77127724. https://doi.org/10.3390/ijerph120707712

Gomes-Mascarenhas, L., Macedo-Salgueirosa, F., FerreiraNunes, G., Martins, P., Stabelini- Neto, A., Campos, W. (2005). Relationship between different rates of physical activity and adiposity predictors in male and female adolescents. Rev Bras Med Esporte 11, 4, 204e-208e

Gomez-Campos, R., Cruz-Flores, I., Mendez-Cornejo, M., Pezoa-Fuentes, P., Urra-Albornoz, C., \& Cossio-Bolaños, MA. (2019). La adiposidad corporal se relaciona con el rendimiento del salto horizontal en niños. Retos: Nuevas Perspectivas de Educacioìn Fì̀sica, Deporte y Recreacioin, 36, 370-375.

Gomez-Campos, R., Arruda, M., Luarte-Rocha, C., Urra Albornoz, C., Almonacid Fierro, A., \& Cossio-Bolaños, M. (2016). Enfoque teórico del crecimiento físico de niños y adolescentes. Revista Española de Nutrición Humana y Dietética, 20(3), 244. https://doi.org/10.14306/ renhyd.20.3.198

Gómez-Campos, R., Vilcazán, É., Arruda, M., Hespañol, J., \& Cossio-Bolaños, M. (2012). Validación de un cuestionario para la valoración de la actividad física en escolares adolescentes. Anales de La Facultad de Medicina, 73(4), 307-314. https://doi.org/https://doi.org/10.15381/ anales.v73i4.1029

Lee, J., Chung, D.-S., Kang, J.-H., \& Yu, B.-Y. (2012). Comparison of visceral fat and liver fat as risk factors of metabolic syndrome. Journal of Korean Medical Science, 27(2), 184-189. https://doi.org/10.3346/ jkms.2012.27.2.184

Ministerio de Salud. (2018). Encuesta Nacional de Salud 2016- 2017. Santiago, Chile. Retrieved from http:// epi.minsal.cl/resultados-encuestas/

Murabito, J. M., Massaro, J. M., Clifford, B., Hoffmann, U., \& Fox, C. S. (2013). Depressive symptoms are associated with visceral adiposity in a community-based sample of middle-aged women and men. Obesity (Silver Spring, Md.), 21(8), 1713-1719. https://doi.org/10.1002/oby.20130

Myrtaj, N., Maliqi, A., Gontarev, S., Kalac, R., Georgiev, G., \& Stojanoska, B. B. (2018). Anthropometry and Body Composition of Adolescents in Macedonia. International Journal of Morphology, 36(4), 1398-1406. https://doi.org/10.4067/S0717-95022018000401398

Núñez-Quiroga, J., Zurita-Ortega, F., Ramírez-Granizo, I., Lozano-Sánchez, A., Puertas-Molero, P., \& Ubago-Jiménez, JL. (2019). Análisis de la relación entre los hábitos físicosaludables y la dieta con la obesidad en escolares de tercer ciclo de Primaria de la Provincia de Granada. Retos: Nuevas Perspectivas de Educacioìn Fì̀sica, Deporte y Recreacioìn, 35, 31-35

Ogden, C. L., Carroll, M. D., Curtin, L. R., McDowell, M. A., Tabak, C. J., \& Flegal, K. M. (2006). Prevalence of Overweight and Obesity in the United States, 1999-2004. JAMA, 295(13), 1549. https://doi.org/10.1001/ jama.295.13.1549

Poitras, V. J., Gray, C. E., Borghese, M. M., Carson, V., Chaput, J.-P., Janssen, I., ... Tremblay, M. S. (2016). Systematic review of the relationships between objectively measured physical activity and health indicators in school-aged children and youth. Applied Physiology, Nutrition, and Metabolism, 41(6(Suppl. 3)), S197-S239. https://doi.org/ 10.1139/apnm-2015-0663

Ross, W. D., Marfell-Jones, M. J., MacDougall, J., Wenger, H., \& Green, H. (1991). Physiological testing of the high performance athlete. (Champaign III, Ed.), Kinanthropometry Champaign IL: Human Kinetics Books (2nd ed.). Ilinois, EEUU: Human Kinetics Books.

Sahoo, K., Sahoo, B., Choudhury, A. K., Sofi, N. Y., Kumar, R., \& Bhadoria, A. S. (2015). Childhood obesity: causes and consequences. Journal of Family Medicine and Primary Care, 4(2), 187-192. https://doi.org/10.4103/22494863.154628

Sepúlveda Cáceres, X., Méndez Cornejo, J., Duarte Farfán, C., Herrera, M., Gómez-Campos, R., Lazari, E., \& CossioBolaños, M. (2018). Relationship between body adiposity and horizontal jump in school children and adolescents. Revista Chilena de Pediatria, 89(6), 701-708. https:// doi.org/10.4067/S0370-41062018005001003

Vargas, R., Bahamonde, C., Cancino, J., Correa, P., Michelow, S., Gatica, P., ... Cossio-Bolaños, M. (2014). Parámetros de crecimiento y adiposidad corporal de adolescentes chilenos a través de la referencia CDC-2000 y CDC-2012: estudio ACECH. Revista Chilena de Nutrición, 41(1), 54-60. https://doi.org/10.4067/S0717-75182014000100007

Wardle, J., \& Boniface, D. (2008). Changes in the distributions of body mass index and waist circumference in English adults, 1993/1994 to 2002/2003. International Journal of Obesity, 32(3), 527-532. https://doi.org/10.1038/ sj.ijo.0803740

Wechsler, H., Devereaux, R. S., Davis, M., \& Collins, J. (2000). Using the School Environment to Promote Physical Activity and Healthy Eating. Preventive Medicine, 31(2), S121-S137. https://doi.org/10.1006/PMED.2000.0649

World Health Organization. (2018). Obesity and overweight. Retrieved April 22, 2019, from https://www.who.int/newsroom/fact-sheets/detail/obesity-and-overweight

Yáñez-Silva, A., Hespanhol, J. E., Gómez-Campos, R., \& Cossio-Bolaños, M. (2014). Valoración de la actividad física en adolescentes escolares por medio de cuestionario. Revista Chilena de Nutrición, 41(4), 360-366. https:/ /doi.org/10.4067/S0717-75182014000400003

Zurita-Ortega, F., Ubago-Jiménez, J. L., Puertas-Molero, P., González-Valero, G., Castro-Sánchez, M., \& Chacón-Cuberos, R. (2018). Niveles de actividad física en alumnado de Educación Primaria de la provincia de Granada. Retos/ : Nuevas Perspectivas de Educacioìn Fiìsica, Deporte y Recreacioìn, 0(34), 218-221. Retrieved from https:// recyt.fecyt.es/index.php/retos/article/view/60098/38735 